\title{
CONTAMINAÇÃO AMBIENTAL POR COMPOSTOS ORGANOESTÂNICOS
}

\author{
Ana Flavia Locateli Godoi \\ Micro and Trace Analysis Center, Chemistry Department, Universitaire Instelling Antwerpen, Universiteitsplein 1, 2610 Wilrijk - \\ Antwerp - Belgium \\ Rodrigo Favoreto e Mary Santiago-Silva* \\ Instituto de Química de Araraquara, Universidade Estadual Paulista, CP 355, 14801-970 Araraquara - SP
}

Recebido em 15/7/02; aceito em 17/4/03

\begin{abstract}
ENVIRONMENTAL CONTAMINATION FOR ORGANOTIN COMPOUNDS. The occurrence and the effects of organotin compounds (OTs) have been studied since a long time, due to their widespread use and deleterious effects. Some OTs are used as pesticides in crops, or as biocides in antifouling paints, applied in the ship hulls to avoid attachment and growth of tube worms, mussels and barnacles. However, "nontarget" organisms may be exposed, resulting in the poisoning of biological system, originating mutations and sentencing species to extinction. In this work we reported a revision study on the history of OTs and the techniques developed for its assessment and control.
\end{abstract}

Keywords: organotin compounds; environment; analytical techniques.

\section{INTRODUÇÃO}

Os efeitos da poluição no mundo têm servido de alerta para a população nos últimos anos. Grandes esforços têm sido feitos na busca de soluções e alternativas para melhorar o quadro ambiental do nosso planeta. É visivelmente crescente o interesse pelo problema da contaminação que afeta a atmosfera, o solo, as florestas, os animais, os rios e os oceanos.

Recentemente observou-se um aumento particular no interesse e na preocupação com a contaminação dos oceanos já que, nas últimas décadas, a influência do homem sobre os ecossistemas marinhos tornou-se muito mais intensa. A maioria dos poluentes entra no ambiente marinho, resultando em efeitos desastrosos como danos aos recursos vivos, riscos à saúde humana, obstáculos às atividades marinhas incluindo a pesca, e prejuízo da qualidade da água. Dentre esses efeitos, incluem-se não apenas os poluentes com propriedades tóxicas mas, também a poluição térmica, microorganismos patogênicos, resíduos sólidos, material suspenso, nutrientes, além de outros tipos de influência sobre o ambiente aquático ${ }^{1}$.

Entre as atividades humanas que podem introduzir substâncias químicas no ambiente marinho está o uso de tintas anti-incrustrantes, aplicadas nos cascos de navios para evitar que algas, mexilhões e outros organismos se agarrem às embarcações e cujo princípio ativo é constituído por compostos organoestânicos - Tributilestanho (TBT) ou Trifenilestanho (TPhT). Um dos principais efeitos do uso de tais substâncias é o envenenamento do sistema biológico, principalmente de moluscos e ostras, originando mutações e condenando espécies à extinção. Estudos sobre a ocorrência e os efeitos destes organometálicos têm sido desenvolvidos principalmente em países do hemisfério norte, como Espanha, França, Inglaterra, Japão e Estados Unidos.

Alguns compostos organoestânicos são também utilizados em lavouras, como pesticidas. O acúmulo de compostos organoestânicos em solos, pode afetar a atividade biológica por mudanças no com-

*e-mail: mssqam@iq.unesp.br portamento de artrópodes terrestres e na microflora, sendo que ambos contribuem para a decomposição do material orgânico e mineralização de nutrientes ${ }^{2}$.

\section{Histórico}

O primeiro estudo sistemático sobre os compostos organoestânicos (OTs, do inglês "organotins") foi realizado por Sir Edward Frankland (1825-1899) que, em 1853, preparou o di-iodeto de dietilestanho e, em 1859, o tetraetilestanho. Estes compostos mantiveram-se por muito tempo apenas sob interesse puramente científico, já que não revelavam nenhum potencial comercial ${ }^{3}$. A primeira citação de aplicação prática foi feita em uma patente apresentada em 1925 , onde eram chamados de "agentes anti-traças", apesar de nunca terem sido usados como tal ${ }^{4}$. Começaram a se destacar por volta de 1940, quando a indústria de plásticos iniciou sua expansão e a importância do cloreto de polivinila (PVC) passou a ser conhecida. Este polímero, sob efeito do calor ou da luz, é degradado com conseqüente descoloramento. Uma pesquisa, feita com compostos que pudessem inibir esta degradação, revelou a poderosa ação estabilizante de alguns derivados dos compostos organoestânicos. Foram então introduzidos para este propósito nos Estados Unidos no final dos anos 40 e na Europa em meados dos anos $50^{3}$.

O uso dos OTs como biocidas surgiu de um estudo sistemático, conduzido por volta de 1950, pelo Conselho Internacional de Pesquisas sobre Tintas, no Instituto de Química Orgânica em Utrecht, sobre a ação desses compostos em fungos e bactérias, organismos marinhos, vermes parasitas, caramujos aquáticos e insetos ${ }^{4}$. As propriedades biocidas dos compostos trialquilestânicos e triarilestânicos foram então descobertas e passaram a ser exploradas comercialmente. Desde o final de 1960, três outros compostos organoestânicos têm sido utilizados como pesticidas, protegendo pomares e uma variedade de outras plantações contra o ataque das pragas. Outras aplicações biocidas dos compostos triorganoestânicos que foram desenvolvidas incluem preservação de madeira e tintas anti-incrustrantes; outras, menos significativas, são desinfetantes e tratamentos algicidas em materiais de construção. Em 1990, o consumo de OTs era estimado em cerca de 35000 toneladas por ano $^{3}$. Estimativas recentes 
assumem que a produção mundial de compostos organoestânicos pode atingir 50000 toneladas por ano.

\section{Propriedades, características e aplicações}

Os OTs são caracterizados pela presença de uma ou mais ligações estanho-carbono e apresentam a fórmula geral $\mathrm{R}_{\mathrm{n}} \mathrm{SnX}_{4-\mathrm{n}}$, onde $\mathrm{R}$ é um grupo alquil ou aril, $\mathrm{X}$ é uma espécie aniônica, como cloreto, óxido, hidróxido ou outro grupo funcional, e n varia de 1 a $4^{3}$.

O número de ligações $\mathrm{Sn}-\mathrm{C}$ tem um grande efeito sobre as propriedades dos OTs, permitindo uma série de aplicações. Os compostos tetraorganoestânicos $\mathrm{R}_{4} \mathrm{Sn}$ não possuem atividade biológica significativa e sua maior aplicação comercial é como precursor para outros compostos organoestânicos. A atividade biológica máxima ocorre na série de OTs onde $\mathrm{n}=3$, e os compostos triorganoestânicos são utilizados comercialmente como biocidas em várias áreas.

$\mathrm{Na}$ agricultura, compostos trialquilestânicos não têm qualquer aplicação apreciável como fungicidas ou acaricidas, em parte devido à alta fitotoxidade e ao fato de que alguns apresentam toxicidade em mamíferos. Além disso, tais compostos são freqüentemente pouco eficazes em experimentos laboratoriais. Já os compostos trifenilestânicos apresentam baixa fitotoxidade e são largamente utilizados como fungicidas 5 .

Os compostos dialquilestânicos $\mathrm{R}_{2} \mathrm{SnX}_{2}$ são poderosos estabilizantes para o PVC; os mais efetivos são aqueles contendo ligações Sn-S, sendo que os bis(carboxilatos) de dialquilestanho são preferidos quando é necessária a resistência à luz e ao calor. Os compostos monoorganoestânicos exibem baixa toxicidade e, portanto, são industrialmente interessantes. Existem indicações de que possam ser utilizados como agentes à prova de água para materiais celulósicos, como algodão têxtil, papel e madeira, e como retardantes de fogo para tecidos de $1 \tilde{a}^{3}$.

\section{TINTAS ANTI-INCRUSTRANTES À BASE DE COMPOSTOS ORGANOESTÂNICOS}

Os cascos dos navios imersos no mar por qualquer período de tempo estão sujeitos ao ataque de organismos incrustrantes como cracas, mexilhões e algas, resultando na diminuição significativa da eficiência operacional com conseqüente aumento nos custos, devido principalmente ao aumento no consumo de combustível. A incrustração de $10 \mu \mathrm{m}$ no casco de um navio pode significar um aumento de 0,3 a $1 \%$ no consumo de combustível ${ }^{6}$. A solução está nas visitas regulares às docas, onde tais organismos podem ser raspados do casco, o qual é então recoberto com uma camada de tinta anti-incrustrante.

Há dois mil anos atrás, os cascos dos navios, que eram feitos de madeira, eram revestidos com chumbo e "untados" com misturas de óleo de baleia, enxofre e arsênio. Em 1625, uma receita letal combinando arsênio, cobre e goma em pó foi patenteada como agente antiincrustrante $^{7}$. O óxido cuproso passou a ser utilizado como biocida em tintas anti-incrustrantes a partir da metade do século XIX. No entanto, as tintas à base de cobre se tornavam ineficientes em menos de um ano e, portanto, biocidas mais efetivos foram necessários ${ }^{8}$. Vários outros biocidas foram testados em tintas, e compostos organomercurosos e arsenicais foram utilizados até por volta de 1970, para aumentar a eficiência das tintas à base de óxido cuproso. Em 1961, foi desenvolvida a primeira tinta incorporando um composto organoestânico como biocida e, devido à sua eficiência e disponibilidade em cores variadas, têm sido amplamente utilizadas até hoje, principalmente aquelas contendo tributilestanho (TBT) ${ }^{9}$. Aplicadas nos cascos das embarcações, essas tintas proporcionam mais de cinco anos de proteção.
As primeiras tintas anti-incrustrantes organoestânicas continham óxido de bis-tributilestanho ou haletos de tributilestanho, e suas formulações apresentavam os compostos TBT simplesmente misturados na tinta; por isso eram chamadas de tintas de livre-associação. Recobrimentos mais efetivos foram desenvolvidos, onde monômeros de TBT eram incorporados com outros monômeros para formar copolímeros, tais como metacrilato de tributilestanho ${ }^{8}$.

As tintas convencionais previnem o crescimento de organismos incrustrantes pela liberação do biocida na superfície da tinta, que depois é varrida pelo atrito da água. A taxa de liberação diminui exponencialmente em um período de meses, até que não exista mais biocida disponível para atingir a superfície da tinta, e os organismos comecem a formar uma camada incrustrada sobre o casco. Esta forma de liberação é ineficiente, pois a quantidade de biocida liberada inicialmente é muito maior que a necessária. Após aproximadamente 1 ano, o filme de tinta envelhece e o carbonato de cálcio $\mathrm{CaCO}_{3}$ entope os microcanais na superfície da tinta, inibindo a liberação do biocida. A superfície então começa a ser incrustrada $a^{6,8}$.

Já as tintas anti-incrustrantes copolímeras, desenvolvidas no início dos anos 70, liberam o biocida a uma taxa sempre constante. $\mathrm{O}$ organoestanho é quimicamente ligado na matriz da tinta, como o copolímero metacrilato de tributilestanho/metil metacrilato. Essa ligação é quebrada na presença de água sob condições levemente básicas, justamente a condição encontrada na água do mar. Conforme o polímero vai sendo quebrado, a superfície da tinta vai sendo desgastada, expondo constantemente novas superfícies que podem liberar mais biocida. Desta maneira, a liberação de TBT é feita pelo movimento da água sobre a superfície da tinta, a qual vai lentamente sendo polida. E como a liberação do biocida é governada pela decomposição química do grupo TBT, ao invés da dissolução da partícula da tinta, a taxa de liberação da tinta copolímera é muito mais lenta que no caso da tinta de livre-associação, e a proteção contra a incrustração pode durar de 5 a 7 anos. As tintas copolímeras podem ser aplicadas no casco do navio sem a necessidade de remover a camada copolímera anterior, o que diminui os custos de manutenção e menos TBT entra no ambiente através da raspagem do casco ${ }^{6}$.

\section{UTILIZAÇÃO DE COMPOSTOS ORGANOESTÂNICOS COMO PESTICIDAS}

A partir de 1960, novos compostos fungicidas contendo estanho foram desenvolvidos e comercializados pelas maiores indústrias químicas multinacionais. A companhia alemã Hoechst (atual Aventis S.A.) produziu e preparou o chamado Brestan, baseado no acetato de trifenilestanho e a Philips Dufan introduziu no mercado o Du-ter, baseado no hidróxido de trifenilestanho, que provou ser efetivo no controle de fungos fitopatogênicos. A Dow Chemical, por sua vez, lançou o acaricida Plictran, em 1967, baseado no hidróxido de tricicloexilestanho (cyhexatin). O grupo Royal Dutch/Shell produziu o Vendex, baseado no óxido de fenbutatina e a alemã Bayer AG produziu o Peropal, baseado no tricicloexilestanho-1,2,4-triazol (azocyclotin). Estas formulações são ainda utilizadas hoje em dia ${ }^{5,10}$.

A aplicação destes compostos organoestânicos no controle de diferentes pragas, em diferentes lavouras, é mostrada na Tabela 1.

A degradação destes pesticidas, dependendo das condições ambientais, pode ser muito lenta. Segundo Müller ${ }^{17}$, a degradação do cyhexatin $\left[\left(\mathrm{C}_{6} \mathrm{H}_{11}\right)_{3} \mathrm{SnOH}\right]$ é de cerca de 50 dias no solo, degradando-se inicialmente a óxido de dicicloexilestanho $\left[\left(\mathrm{C}_{6} \mathrm{H}_{11}\right)_{2} \mathrm{SnO}\right]$, posteriormente a ácido cicloexilestanônico $\left[\left(\mathrm{C}_{6} \mathrm{H}_{11}\right) \mathrm{SnO}_{2} \mathrm{H}\right] \mathrm{e}$, finalmente, chegando a óxido de estanho $\left(\mathrm{SnO}_{2}\right)^{18,19}$. Já em fase aquosa, a concentração de cyhexatin decresce rapidamente nos primeiros 57 dias, quando é observado um equilíbrio entre o cyhexatin do sedimento e o da fase orgânica sob o sedimento. Após poucos dias, os metabólitos 
Tabela 1. Utilização de compostos organoestânicos como pesticidas em lavouras

\begin{tabular}{|c|c|c|c|}
\hline Pestidida & Culturas & Pragas & Ref. \\
\hline \multirow[t]{3}{*}{ Cyhexatin } & Citrus & Tetranychus urticae, Phyllocoptruta oleivora e Brevipalpus phoenicis & $11-13$ \\
\hline & Maçã & Panonychus ulmi & $14-16$ \\
\hline & Beringela & Tetranychus urticae & \\
\hline \multirow{7}{*}{$\begin{array}{l}\text { Cyhexatin, óxido } \\
\text { de fenbutatina e } \\
\text { azocyclotin }\end{array}$} & Citrus & $\begin{array}{l}\text { Brevipalpus phoenicis, Phyllocoptruta oleivora, Panonychus citri e } \\
\text { Polvphagotarsonemus latus }\end{array}$ & 5 \\
\hline & Batatas & Phytophthora infestans & \\
\hline & Amendoim & Cercospora arachidicola e Cercosporium personatum & \\
\hline & Soja & C. kikuchi, Diaporthe phaseolorum, C. dematium e Golpearia glycines & \\
\hline & Beterraba & C. beticola, Ersiphe betae, Phoma betae e Phythium aphanidermatum & \\
\hline & Café & P. syringae & \\
\hline & Cacau & Cacao moniliasis e Phytophthora palmivora & \\
\hline
\end{tabular}

do azocyclotin, estão distribuídos entre a fase aquosa, biota e sedimento.

A sedimentação do azocyclotin $\left[\left(\mathrm{C}_{6} \mathrm{H}_{11}\right)_{3} \mathrm{Sn}-1,2,4\right.$-triazol], por sua vez, é relativamente rápida em ambiente aquático (meio no qual estão preferencialmente concentrados seus metabólitos): logo após a aplicação, são iniciados os processos de solubilização e de metabolização, onde o composto é rapidamente hidrolisado à cyhexatin, depois a óxido de dicicloexilestanho, ácido cicloexilestanônico e, finalmente, chegando a óxido de estanho.

$\mathrm{O}$ óxido de fenbutatina $-\left\{\left[\left(\mathrm{C}_{6} \mathrm{H}_{5}\right)-\mathrm{C}\left(\mathrm{CH}_{3}\right)_{2} \mathrm{CH}_{2}\right]_{3} \mathrm{Sn}\right\}_{2} \mathrm{O}$ degrada-se inicialmente a estanato de di-hidroxi-bis(2-metil-2-fenilpropil) - $\left[\left(\mathrm{C}_{6} \mathrm{H}_{5}\right)-\mathrm{C}\left(\mathrm{CH}_{3}\right)_{2} \mathrm{CH}_{2}\right]_{2} \mathrm{Sn}(\mathrm{OH})_{2}$ - e, posteriormente, a ácido 2-metil2-fenilpropil estanônico - $\left[\left(\mathrm{C}_{6} \mathrm{H}_{5}\right)-\mathrm{C}\left(\mathrm{CH}_{3}\right)_{2} \mathrm{CH}_{2}\right] \mathrm{SnO}_{2} \mathrm{H}^{20,21}$.

\section{TOXICIDADE E IMPACTO AMBIENTAL DOS COMPOSTOS ORGANOESTÂNICOS}

A preocupação a respeito do impacto ambiental causado pela utilização de tintas anti-incrustrantes à base de OTs surgiu no início dos anos 80, quando ficou claro que os organismos que aderem nos cascos dos navios não eram as únicas vítimas, ou seja, outros animais e plantas eram expostos. Um exemplo foi o desenvolvimento de conchas anormalmente finas em ostras presentes em águas contaminadas com os agentes anti-incrustrantes.

Do ponto de vista de poluição ambiental, o grande problema é quando as embarcações estão paradas nos ancoradouros. Um aumento significativo dos OTs pode ocorrer nas águas de atracadouros e marinas contendo grande número de pequenas embarcações de passeio, a maioria pintada com o sistema antigo e convencional, ou seja, tintas de livre-associação à base de OTs.

Além dos efeitos observados nas culturas de ostras, vários estudos demonstraram os efeitos tóxicos dos compostos organoestânicos em outras espécies marinhas como moluscos, algas e zooplânctons sob concentrações de poucos nanogramas por litro $^{22}$. Entre os mais significativos, estão os efeitos do TBT sobre a reprodução de ostras e neogastrópodes, e indicam que o composto está entre os mais tóxicos já introduzidos propositalmente no ambiente aquático ${ }^{23}$.

Em 1981, o surgimento de órgãos sexuais masculinos em fêmeas de caramujos (Nassarius obsoletus) foi relacionado à contaminação por TBT. Esse fenômeno foi chamado de "imposex" ou "pseudohermafroditismo". O grau de desenvolvimento do pênis e a frequiência do "imposex" foram relacionados aos níveis de TBT e foram mais intensos próximos de portos e marinas ${ }^{23}$. Estudos de campo feitos posteriormente confirmaram a relação entre o "imposex" nos caramujos (Ilyanassa obsoleta) e o $\mathrm{TBT}^{24}$. A toxicidade dos compostos organoestânicos varia consideravelmente de acordo com o número e a natureza dos grupos orgânicos e, geralmente, diminui na ordem tri- $\left(\mathrm{R}_{3} \mathrm{SnX}\right)>$ di- $\left(\mathrm{R}_{2} \mathrm{SnX}_{2}\right)>$ mono- $\left(\mathrm{RSnX}_{3}\right)$ substituídos, enquanto os tetraorganoestânicos $\mathrm{R}_{4} \mathrm{Sn}$ apresentam baixa toxicidade ${ }^{25}$. $\mathrm{O}$ aumento na toxicidade pode ser relacionado à insolubilidade dos compostos em água ${ }^{26}$. A hidrofobicidade, processo de partição regido termodinamicamente entre a água e a fase lipídica do organismo é, de fato, considerada o principal parâmetro na bioconcentração desses compostos ${ }^{23}$.

Várias pesquisas têm sido feitas com o objetivo de se avaliar os efeitos dos OTs nos organismos vivos. Estudos com algas mostram que o tipo de resposta depende da espécie e da dose do composto, mas essa resposta geralmente envolve taxas de crescimento e de fotossíntese reduzidas, e até a morte das células. Dos compostos organoestânicos mais largamente utilizados, o tributil e o trifenilestanho aparecem como os mais tóxicos para as microalgas marinhas e de água doce. Vários estudos mostraram que as microalgas se adaptam aos OTs e são capazes de acumular alguns desses compostos em baixas concentrações, próximas àquelas que ocorrem em águas naturais ${ }^{27}$. Os bivalves, organismos filtradores, apresentam grande potencial de bioacumulação dos OTs e, juntamente com moluscos e ostras, servem como bioindicadores da poluição por TBT nos ecossistemas marinhos por possuírem habilidade limitada para metabolizar e eliminar tal composto. Essas observações alertam para o risco ambiental potencial relacionado à biotransferência desses compostos aos outros elos da cadeia alimentar, podendo alcançar o homem ${ }^{23}$.

Com relação ao impacto ambiental dos OTs utilizados como defensivos agrícolas, observou-se grande tendência de acumulação. Devido à sua baixa solubilidade em água, o cyhexatin (principal metabólito) foi sorvido pelo sedimento e pela camada da biota em contato direto com o sedimento ${ }^{28}$.

Os efeitos do pesticida azocyclotin foram estudados em ambiente de água doce ${ }^{29}$. Os resultados mostraram que sob concentrações nominais de $135 \mu \mathrm{g} \mathrm{L} \mathrm{L}^{-1}$, o azocyclotin provocou severos efeitos na comunidade plantônica, incluindo fito- e picoplânctons e também algas. Para os zooplânctons, os efeitos foram observados sob concentrações nominais de $45 \mu \mathrm{g} \mathrm{L} \mathrm{L}^{-1}$. Nos sedimentos, detectou-se um aumento na atividade de degradação microbiana anaeróbia provocando alta mortalidade de plânctons.

\section{TENDÊNCIAS ATUAIS E FUTURAS}

A evidência dos efeitos nocivos dos compostos triorganoestânicos sobre a reprodução e o crescimento das várias formas de vida marinha tem incentivado vários países a regulamentar ou banir o uso de produtos anti-incrustantes. Como consequiência dessas restrições, a busca por produtos alternativos tem crescido bastante nos últimos anos. 
Uma das alternativas tem sido a utilização de produtos baseados principalmente em óxidos de cobre juntamente com biocidas orgânicos intensificadores ("boosters"), tais como triazinas, isotiazolonas, ditiocarbamatos, para aumentar a eficiência da formulação ${ }^{30}$.

A "Kyosie Chemical Company of Tokyo" propôs a formulação de novos agentes anti-incrustrantes à base de compostos hexacianos, que não apresentam taxa de liberação intensa, mas que mantêm a atividade anti-incrustrante por longo tempo. Também estão em estudo compostos hexacianoferratos ${ }^{31}$.

Um novo protocolo analítico demonstra todos os fatores envolvidos na ação de erosão e anti-incrustração, revelando as reações químicas envolvidas. O óxido cuproso apresenta influência regulatória sobre a penetração da água e a conseqüente hidrólise da tinta. A proposta é de uma tinta anti-incrustrante biodegradável, eficiente por pelo menos 22 meses $^{32}$.

Alternativas mais "ecológicas" também têm surgido, como a pesquisa do Prof. R. Crespo da Universidade Federal Fluminense que, juntamente com seus colaboradores, isolou um componente químico das algas vermelhas que pode substituir o TBT. O trabalho já vem sendo feito há 5 anos e está em fase de testes da substância frente aos incrustantes ${ }^{33}$.

\section{RELEVÂNCIA DO TEMA NO CONTEXTO AMBIENTAL}

A utilização dos compostos organoestânicos cresceu nos últimos 30 anos de cerca de 1500 para 50000 toneladas por ano, e esses compostos atualmente são empregados em uma grande variedade de indústrias. Um grande número de trabalhos de pesquisa publicados sobre esses compostos tratam do seu uso e isso inclui estudos extensivos sobre sua toxicologia. E quando olhamos para o futuro ambiental desses compostos, devemos levar em conta o aumento abrupto da quantidade introduzida no ambiente e o desenvolvimento de novos compostos organoestânicos com potencial industrial, cujas características e possíveis efeitos precisam ser avaliados.

No entanto, todos os estudos feitos enfocando os compostos organoestânicos referem-se às regiões norte e oeste dos oceanos Atlântico e Pacífico, não sendo possível encontrar na literatura nenhum estudo enfocando regiões tropicais e sub-tropicais, como o Atlântico Sul. Portanto, o desenvolvimento de novas pesquisas é de extrema importância para se conhecer a exata extensão dos impactos ambientais provocados por esses compostos, principalmente no Brasil, onde não existe nenhum tipo de controle ou legislação que gerencie esse problema.

Os sedimentos marinhos normalmente são o primeiro alvo de estudo pois os compostos organoestânicos sofrem pouca dispersão, sendo acumulados muito próximos ao local onde foram lançados. Sob determinadas condições, esses contaminantes podem ser liberados do sedimento para a água ou acumulados na cadeia alimentar. Conseqüentemente, os sedimentos podem ser considerados tanto absorvedores como fontes dos contaminantes no ambiente aquático, o que os coloca no centro da questão ambiental, com implicações científicas importantes.

\section{LEGISLAÇÃO PARA OS COMPOSTOS ORGANOESTÂNICOS}

As primeiras ações regulatórias e legislativas de gerenciamento de riscos para o tributilestanho foram adotadas na França em 1982, seguida pelo Reino Unido em 1985. Essas ações foram baseadas em uma avaliação dos impactos do TBT sobre ostras comercialmente cultivadas (Crasostrea gigas). Um decréscimo significativo na abundância das ostras que habitavam a Baía de Arcachon na costa Atlântica da França foi observado no final dos anos 70. Além disso, a ocorrência de má formação das conchas, o que raramente era obser- vado antes, aumentou afetando 80-100\% dos animais. Em 1980, o TBT proveniente de barcos de lazer nas vizinhanças do cultivo das ostras foi suspeito de ser o agente causador deste efeito. Em 1982 os franceses baniram a aplicação de tintas anti-incrustantes contendo TBT em cascos de barcos menores de $25 \mathrm{~m}$. O fato é que os barcos pequenos ficam muito tempo nos atracadouros, muito próximos da costa, onde o TBT se acumula no sedimento, enquanto que os grandes barcos e navios passam a maior parte do tempo em alto mar, resultando na diluição do biocida em um grande volume de água. Barcos de alumínio foram excluídos porque a aplicação do agente anti-incrustante alternativo, o cobre, poderia causar severa corrosão. Após vários anos, os cientistas franceses notaram uma diminuição significativa nas concentrações de OTs nas águas da Baía de Arcachon. Também documentaram um aumento significativo na produção de ostras e diminuição na deformação das conchas ${ }^{8,25}$.

Os britânicos, similarmente, observaram os impactos sobre as populações de ostras que habitavam áreas de intensa atividade náutica. $\mathrm{O}$ governo introduziu, em 1985, regulamentações para impedir a venda ao varejo das tintas com TBT que liberavam o biocida em altas taxas. Essas regulamentações proibiram a venda de tintas de TBT contendo mais de 7,5\% de estanho total em copolímeros ou 2,5\% de estanho total como estanho livre, ou com uma taxa de liberação maior que $4,0 \mu \mathrm{g} \mathrm{cm}^{-2} \mathrm{dia}^{-1}$. Também estabeleceram uma concentração limite na água de $20 \mathrm{ng} \mathrm{L}^{-1}$ com o objetivo de proteger seus recursos marinhos. Em 26 de fevereiro de 1987, oficiais do Departamento de Meio Ambiente Britânico determinaram a redução da concentração limite para $2 \mathrm{ng} \mathrm{L}^{-1}$ e a suspensão do fornecimento de tintas anti-incrustantes contendo TBT para o uso em barcos menores de $25 \mathrm{~m}^{8,25}$.

Em 1996, o Comitê de Proteção do Ambiente Marinho das Nações Unidas (MEPC) redigiu uma proposta sugerindo retirada gradual das tintas a base de TBT nos próximos 10 anos, culminando no banimento em 2006, apesar dos apelos de vários grupos ambientais e de certos países como o Japão (que baniu a tinta desde 1992 nos cascos de seus navios), que pedem a retirada imediata de tais biocidas, propondo o banimento para $2003^{34}$. Entretanto, ainda existem dúvidas com respeito à questionável eficácia das alternativas disponíveis ao TBT, ao possível impacto ambiental provocado pelas mesmas, e os enormes custos à indústria naval no caso de um banimento prematuro. Devido à falta de consenso, em 1998 foi estabelecido um grupo de trabalho para discutir tais dúvidas e tentar elucidar e responder às questões ambientais.

Poucos países regulamentam o destino dos resíduos resultantes da limpeza dos cascos dos navios. Estima-se a produção de 0,8 bilhões de litros de resíduos de água contaminada, 2,3 milhões de toneladas de resíduo de tintas e 1,8 milhões de latas de tintas esperando por um destino. Além disso, a cada pintura são liberados solventes na atmosfera contendo Compostos Orgânicos Voláteis ("Volatile Organic Compounds", VOCs) e Poluentes Atmosféricos Perigosos ("Hazardous Air Pollutants", HAPs), que contribuem para a destruição da camada de ozônio ${ }^{35}$.

Não há registros de estudos feitos sobre esses impactos provocados pelos compostos organoestânicos em tintas anti-incrustrantes no Brasil e, portanto, não existe nenhuma regulamentação ou legislação que controle a utilização desses compostos em embarcações no território nacional.

A Figura 1 apresenta as estruturas químicas dos compostos organoestânicos atualmente liberados para comercialização no Brasil ${ }^{36}$.

\section{TÉCNICAS DE ANÁLISE PARA OS COMPOSTOS ORGANOESTÂNICOS}

Uma grande variedade de técnicas analíticas têm sido desenvolvidas para a especiação de compostos organoestânicos, e a maioria 


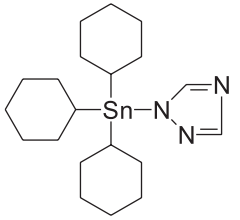

(a)

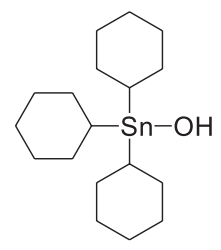

(b)

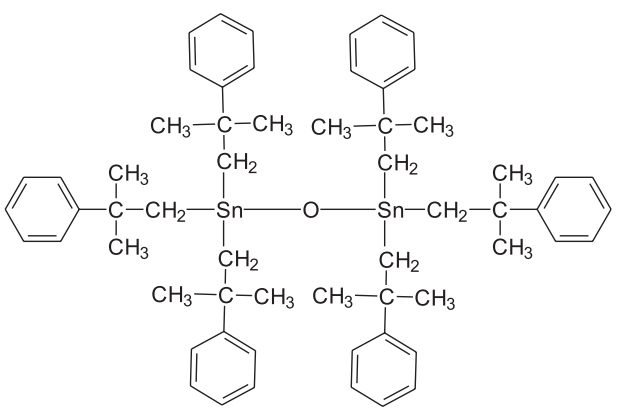

(c)

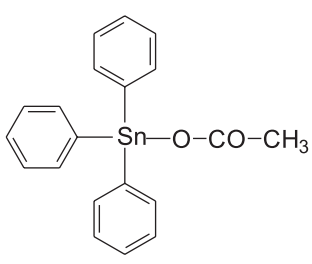

(d)

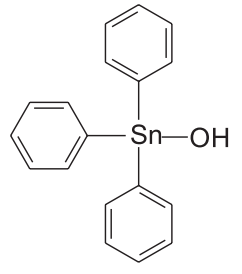

(e)
Figura 1. Pesticidas organoestânicos comercializados no Brasil: (a) Azocyclotin, (b) Cyhexatin, (c) Vendex, (d) Brestan, (e) Duter

dos programas de monitoramento têm dispensado bastante atenção à análise de sedimento e biota, já que estes permitem a avaliação da poluição mais antiga ${ }^{37}$. Embora técnicas de cromatografia líquida venham sendo desenvolvidas ${ }^{38-41}$, os métodos mais amplamente utilizados são baseados na cromatografia a gás pois apresentam maior resolução, maior versatilidade de detecção, e permitem a determinação simultânea de compostos butil-, fenil-, cicloexil-, metil-, etil-, e octilestânicos ${ }^{42,43}$. Outra vantagem da cromatografia a gás sobre a líquida é a possibilidade da utilização de vários padrões internos (P.I.s) e "surrogates" (os padrões empregados como "surrogates" são utilizados para corrigir os valores de concentração dos analitos, devido a perdas durante a manipulação, à ineficiência da extração, à conversão incompleta durante a derivação, às perdas por evaporação etc. $\mathrm{O}$ enriquecimento de sedimentos é usualmente feito em amostras secas, onde, para estimular a adsorção natural e evitar que as substâncias enriquecedoras sejam adsorvidas apenas na superfície da amostra, a forma original da matriz deve ser reconstituída), que permitem que as etapas do procedimento analítico (como quantificação e recuperação) sejam monitoradas. A principal desvantagem dos métodos de cromatografia a gás é que estes usualmente requerem a produção de derivados organoestânicos voláteis para proceder à separação $0^{37}$. Os compostos organoestânicos mono-, di- e trissubstituídos não são voláteis e algumas formas de derivação são usualmente necessárias. A técnica mais amplamente utilizada é a formação de um derivado tetrassubstituído via reação de "Grignard" (brometo ou cloreto de etil, butil ou pentilmagnésio) ${ }^{44-46}$. Além das reações de alquilação, a geração de hidreto tem sido amplamente utilizada para formar deri- vados voláteis, sendo o boroidreto de sódio o mais freqüientemente utilizado ${ }^{47-49}$. Do ponto de vista de detecção, a cromatografia a gás é altamente versátil. A esse respeito, os seguintes detectores têm sido utilizados para a especiação de OTs : detecção por ionização de chama ("Flame Ionization Detection", FID), detecção por captura de elétrons ("Electron Capture Detection", ECD), espectrometria de absorção atômica ("Atomic Absorption Spectrometry", AAS), detecção fotométrica de chama ("Flame Photometric Detection", FPD), detecção por emissão atômica ("Atomic Emission Detection", AED) e espectrometria de massas ("Mass Spectrometry Detection", MSD).

A Tabela 2 resume as informações mais relevantes sobre os procedimentos analíticos utilizados na análise de compostos organoestânicos.

\section{TRATAMENTO DAS AMOSTRAS}

\section{Extração}

Os compostos organoestânicos são encontrados no ambiente aquático associados a uma variedade de contra-íons (carbonatos, cloretos, sulfatos, sulfetos, hidróxidos e biopolímeros) ou como óxidos, e podem interagir com matrizes abióticas em diferentes formas (iônica e/ou hidrofóbica). Os procedimentos analíticos para a especiação dos OTs geralmente visam preservar apenas o radical orgânico durante a extração, enquanto o contra-íon e outros ligantes são eliminados.

No caso dos sedimentos, como os compostos organoestânicos não estão envolvidos em processos mineralógicos e sim ligados apenas na superfície, a dissolução completa da matriz não é considerada necessária. O procedimento básico para a liberação dos OTs do sedimento envolve a lixiviação ácida, sendo os ácidos clorídrico, acético e bromídrico os mais comumente utilizados. A extração é feita por ultra-som, agitação mecânica ou por "soxhlet", com solvente orgânico. O ultra-som tem sido amplamente utilizado devido ao menor tempo de extração e utilização de menor volume de solvente quando comparado às técnicas convencionais.

A combinação de um solvente orgânico de baixa a média polaridade com um ácido complexante (como o ácido acético) caracteriza mais de $50 \%$ dos procedimentos de extração utilizados. Solventes como hexano, tolueno ou diclorometano (DCM) são comumente empregados. Um solvente de polaridade elevada é necessário para favorecer a extração dos OTs mais polares como o $\mathrm{MBT}^{59}$.

\section{Agentes complexantes}

A adição de agentes complexantes é essencial para o aumento da eficiência de extração, especialmente para compostos alquilados de estanho di- e monossubstituídos ${ }^{60}$. A tropolona, em uma variedade de solventes não-próticos (diclorometano, benzeno, éter dietílico, tolueno e hexano), tem sido amplamente utilizada ${ }^{37}$. Os carbamatos também apresentam bom desempenho, embora sejam menos utilizados. Entre eles estão ácido dietilditiocarbâmico (DDTC), pirrolidinoditiocarbamato de amônio (APDC) e dietilditiocarbamato de sódio (NaDDC). A ditizona é mais raramente utilizada.

A tropolona apresenta a vantagem de ser estável em solventes orgânicos, enquanto que os carbamatos têm que ser preparados antes do uso. No entanto, o uso da tropolona na extração de OTs com solventes líquidos a partir de matrizes bióticas e abióticas aumenta também a solubilidade de compostos que são co-extraídos, o que torna a purificação indispensável antes da análise cromatográfica ${ }^{37,60}$. Estudos feitos em presença de tolueno e ácido acético mostraram que a tropolona foi mais eficiente que o NaDDC para os compostos butílicos e para o MPhT, mas com diminuição na eficiência de extra- 
Tabela 2. Procedimentos analíticos utilizados para a análise de compostos organoestânicos em diferentes amostras ambientais

\begin{tabular}{|c|c|c|c|c|}
\hline Amostra & Analitos $^{\mathrm{a}}$ & Técnica de determinação & Comentários & Ref. \\
\hline tecido de salmão & TBT, DBT & GC-FPD $(610 \mathrm{~nm})$ & formação de derivados hidretos & 50 \\
\hline $\begin{array}{l}\text { água, sedimento e } \\
\text { biota marinhos }\end{array}$ & TBT & GC-FPD e GC-MS & $\begin{array}{l}\text { extração com } \mathrm{HCl} \text { e derivação } \\
\text { com "Grignard" }\left(\mathrm{CH}_{3} \mathrm{MgCl}\right)\end{array}$ & 51 \\
\hline água & TBT, DBT & GC-FPD & $\begin{array}{l}\text { extração em discos } \mathrm{C}_{18} \text { "Empore”, } \\
\text { com fluido supercrítico de } \mathrm{CO}_{2}\end{array}$ & 52 \\
\hline sedimento & TBT & GC-FPD (“on-column”) & extração com fluido supercrítico & 53 \\
\hline- & Sn (IV) e monoalquilados & GC-AAS & derivação in situ com $\mathrm{NaBEt}_{4}$ & 54 \\
\hline tecido de atum & TBT, DBT, MBT e TPhT & GC-ICP-AES & extração com forno de microondas & 55 \\
\hline sedimento & MBT, DBT, TBT e TPhT & GC-MS e GC-AES & foram encontrados mais 19 derivados & 45 \\
\hline água & $\begin{array}{l}\text { MBT, DBT, TBT, } \\
\text { MPhT e DPhT }\end{array}$ & GC-FPD & extração por SPME & 56 \\
\hline água e sedimento & MBT, DBT, TBT & GC-FID & extração por SPME e derivação com $\mathrm{NaBEt}_{4}$ & 57 \\
\hline $\begin{array}{l}\text { água de rio, água } \\
\text { residual, areia, ostras }\end{array}$ & $\begin{array}{l}\text { MBT, DBT TBT, DPhT, } \\
\text { MPhT, TPhT, TcHexT, } \\
\text { DOcT, TOcT }\end{array}$ & GC-PFPD & $\begin{array}{l}\text { extração com metanol-HCl em ultra-som } \\
\text { (ostras); com ácido etanóico glacial por } \\
\text { agitação (sedimentos) }\end{array}$ & 49 \\
\hline água & TBT & ICP-TOFMS & SPME ("headspace" de matrizes cloradas) & 58 \\
\hline
\end{tabular}

a MBT $=$ monobutilestanho; DBT $=$ dibutilestanho; $\mathrm{TBT}=$ tributilestanho; MPhT $=$ monofenilestanho; DPhT $=$ difenilestanho; $\mathrm{TPhT}=$ trifenilestanho; $\mathrm{DOcT}=$ dioctilestanho; $\mathrm{TOcT}=$ trioctilestanho; $\mathrm{TcHexT}=$ triciclohexilestanho

ção para o TPhT. Isso pode ser explicado pela falta de efeito dos agentes complexantes sobre os acetatos que já estão solubilizados no tolueno utilizado durante a extração, ou ainda por processos de degradação que levam à formação de uma molécula de DPhT e uma de estanho inorgânico ${ }^{59}$.

Com base nesses resultados, pode-se inferir que, na verdade, a eficiência de extração destes agentes complexantes não é elevada o suficiente para compensar as perdas por degradação e, portanto, muitas vezes sua utilização é descartada.

Existem sugestões de que compostos que possam formar uma espécie de tampão por efeito de íons comuns na extração, como o acetato de sódio por exemplo, possam substituir os agentes complexantes $^{61}$.

\section{Etapas pós-extração}

Quando o ácido acético (HAc) é utilizado na extração, a remoção do ácido da mistura extratora é essencial. Entretanto, a rotaevaporação pode ser dificultada, e o ácido remanescente no extrato pode afetar a reação de derivação. Em vários experimentos, a partição do extrato é feita com água Milli-Q, para remover o HAc da mistura extratora. No entanto, a análise da fase aquosa acética obtida da extração de um sedimento enriquecido com compostos butílicos e fenílicos de estanho mostrou que cerca de $50 \%$ do MBT foi carregado para a fase aquosa ${ }^{59}$.

A utilização de uma solução aquosa de $0,5 \%$ de pirrolidinoditiocarbamato de amônio (APDC), ao invés de somente água, foi testada com êxito. Acredita-se que a APDC forma complexos neutros com os OTs, o que aumenta sua partição para a fase do solvente. A extração líquido-líquido do extrato tolueno-HAc com uma solução aquosa de APDC é recomendada, portanto, para remover o ácido ${ }^{59}$. Sua utilização pode compensar o insucesso dos agentes complexantes, pois diminui as perdas dos analitos.

Outro problema observado na análise de compostos organoestânicos é a interferência dos compostos sulfurados. O enxofre, em seu estado elementar, ocorre em sedimentos anóxicos devido às ati- vidades microbiológicas que convertem sulfatos e sulfetos em enxofre elementar. Esse enxofre é co-extraído com os OTs e também é alquilado na derivação com o reagente de "Grignard", levando à formação de mono-, di- e trisulfetos dialquilados. Mesmo utilizando-se o filtro de $610 \mathrm{~nm}$ na análise por GC-FPD ("Gas Chromatography with Flame Photometric Detection" - cromatografia a gás com detecção fotométrica de chama) ou no modo SIM ("Selected Ion Monitored" - monitoramento do íon selecionado) por GC/MSD ("Gas Chromatography with Mass Spectrometry Detection“ - cromatografia a gás com detecção por espectrometria de massas), altas concentrações de compostos contendo enxofre podem afetar a quantificação de derivados de OTs propilados, pentilados ou hexilados devido aos tempos de retenção coincidentes ${ }^{62,63}$.

Técnicas de detecção baseadas em absorção ou emissão atômica (AAS, ICP, AED) acopladas à cromatografia a gás não sofrem tais interferências por enxofre devido à sua elevada seletividade, mas como os tempos de retenção são coincidentes, a co-eluição de grandes quantidades de compostos organosulfurados leva à uma sobrecarga da fase estacionária, ou ao deslocamento do tempo de retenção, que termina pela identificação errônea dos analitos ${ }^{63}$. Portanto, um método efetivo para a eliminação quantitativa de compostos sulfurados é essencial para a determinação exata dos OTs.

\section{Técnicas de dessulfuração}

Três técnicas de dessulfuração já foram registradas na literatura e adaptadas à análise de OTs, (a) retenção seletiva do enxofre em uma coluna empacotada com cobre em pó ativado, (b) adsorção de enxofre por uma amálgama de mercúrio e (c) precipitação de enxofre por sulfito de tetrabutilamônio. No entanto, com todas essas técnicas, apenas o enxofre elementar pode ser removido, e os alquilsulfetos interferentes permanecem no extrato ${ }^{62}$.

A eliminação de compostos sulfurados por cromatografia de troca de ligante vem sendo utilizada recentemente e baseia-se na formação de ligações coordenativas entre o enxofre e íons metálicos utilizando-se sais de mercúrio, cobre, zinco ou outros. Na cromato- 
grafia de adsorção com sílica-gel recoberta com 25\% de $\mathrm{AgNO}_{3}$, todos os compostos sulfurados interferentes do extrato de sedimento derivado (neste caso um sedimento de marina com grandes quantidades de enxofre elementar) foram removidos quantitativamente. No entanto, apenas os compostos butílicos de estanho foram recuperados, os fenílicos não foram eluídos ${ }^{62}$. Portanto, este método não é completamente útil para a análise de OTs, já que os compostos fenílicos de estanho são adsorvidos irreversivelmente sobre a sílica$\mathrm{AgNO}_{3}$.

Escobar e colaboradores ${ }^{63}$ propuseram um método baseado na oxidação de todas as espécies de enxofre presentes com dimetildioxirano (DMD), formando sulfonas ou óxidos de enxofre. Enquanto as sulfonas são facilmente eliminadas pela purificação com alumina, devido à sua polaridade maior que os OTs, os óxidos de enxofre são evaporados espontaneamente. A quimioseletividade do DMD favorece a oxidação dos compostos de enxofre em sulfonas (Figura 2) em poucos minutos, enquanto que os OTs permanecem sem reagir. Além disso, o excesso de DMD é facilmente removido pela evaporação sob fluxo de $\mathrm{N}_{2}$ antes da purificação. Sua aplicação em análises de rotina apresenta ainda outras vantagens: fácil preparação em solução de acetona e alta reatividade em fase orgânica (sem necessidade de extração líquido-líquido).

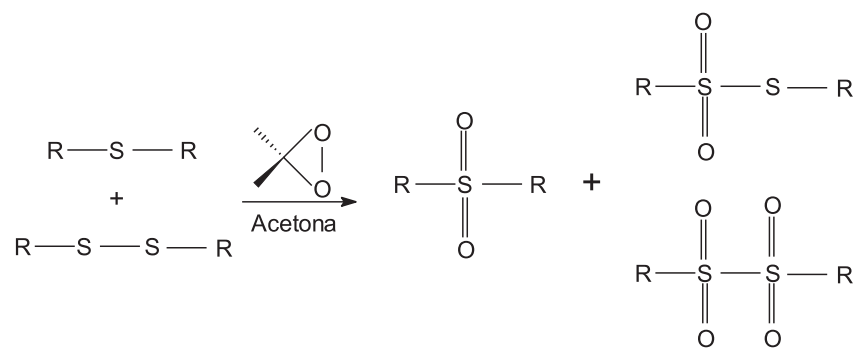

Figura 2. Oxidação dos compostos de enxofre por dimetildioxirano (DMD)

A utilização do DMD em combinação com a purificação com alumina permite a quantificação de todos os analitos e um significante ganho na razão sinal/ruído, aumentando tanto a precisão quanto os limites de detecção na determinação dos OTs ${ }^{63}$.

Os dioxiranos (Figura 3) são peróxidos cíclicos de 3 membros, oxidantes bastante seletivos e suaves frente aos produtos de oxidação. Sua principal característica é a capacidade de transferir um átomo de oxigênio a uma variedade de compostos contendo elétrons $\pi$ ou pares de elétrons não compartilhados. Aparentemente, a fraqueza da ligação O-O, aliada à tensão do anel de três membros, torna este processo particularmente favorável ${ }^{64}$.

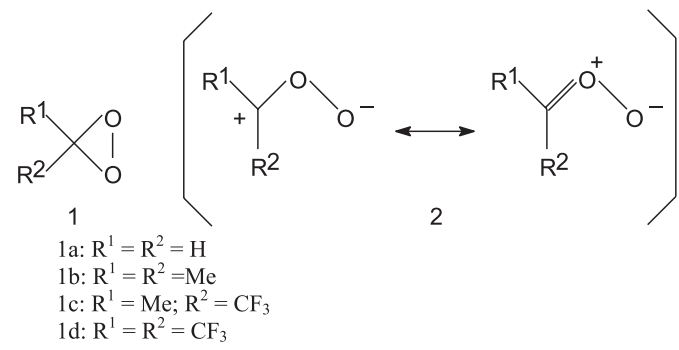

Figura 3. Molécula de dimetildioxirano (DMD) e suas estruturas de ressonância

Estes compostos são facilmente preparados a partir de substâncias comercialmente disponíveis, sendo os métodos mais comuns os que envolvem a oxidação de cetonas por perácidos, particularmente o ácido monoperoxissulfúrico ${ }^{64,65}$.

\section{Derivação}

A cromatografia a gás geralmente requer uma reação de derivação para produzir compostos organoestânicos voláteis e proceder assim à sua separação. Os métodos de conversão dos alquilestanho iônicos em espécies analisáveis por cromatografia a gás incluem: hidridização in situ utilizando $\mathrm{NaBH}_{4}$ ou adição de etila com $\mathrm{NaBEt}_{4}$; derivação utilizando reagentes de "Grignard" (brometos / cloretos de metil-, etil-, propil-, pentil- ou hexilmagnésio), que compreende $53 \%$ dos procedimentos utilizados ${ }^{37}$; e halogenação.

A geração de hidreto (Figura 4) e a adição de etila com $\mathrm{NaBEt}_{4}$ são mais adequadas para amostras aquosas, podendo ser diretamente aplicadas nas amostras. A extração simultânea com a derivação é possível, reduzindo o número de etapas analíticas e, portanto, as fontes de erros. No caso de amostras mais complexas, como sedimentos e biota, ocorrem várias interferências, o que resulta em baixo rendimento e baixa reprodutibilidade ${ }^{66}$.

$$
\begin{gathered}
\mathrm{R}_{\mathrm{n}} \mathrm{Sn}^{(4-\mathrm{n})^{+}}+\mathrm{NaBH}_{4}+\mathrm{H}^{+} \rightarrow \mathrm{R}_{\mathrm{n}} \mathrm{SnH}_{(4-\mathrm{n})}+\mathrm{H}_{2} \uparrow \\
\text { onde } \mathrm{R}=\text { grupo orgânico e } \mathrm{n}=\text { varia de } 1 \text { a } 3
\end{gathered}
$$

\section{Figura 4. Reação de geração de hidreto}

A reação de alquilação de "Grignard" (Figura 5) processa-se quantitativamente levando a derivados estáveis quando é realizada sob condições anidras e com o solvente adequado ${ }^{67}$; quando se utiliza solvente de maior polaridade, grandes quantidades de ácido remanescentes da extração (já que solventes mais polares dissolvem uma parte da fase aquosa que contém o ácido) permanecem mesmo depois da evaporação do extrato o que, provavelmente, afeta negativamente as reações de alquilação, provocando recuperações mais baixas $^{60,66}$. A adição de grupos hexila e pentila têm a vantagem de proporcionar compostos derivados com volatilidade relativamente baixa, o que facilita a etapa de pré-concentração (que geralmente é feita por evaporação com fluxo de $\mathrm{N}_{2}$ ), além de serem compostos que ainda não foram introduzidos no ambiente. O tempo de reação, a temperatura e a agitação da amostra durante a reação não afetam os produtos da derivação ${ }^{68}$.

$$
\mathrm{R}_{\mathrm{n}} \mathrm{Sn}^{(4-\mathrm{n})^{+}}+\mathrm{R}^{\prime} \mathrm{MgX} \rightarrow \mathrm{R}_{\mathrm{n}} \mathrm{SnR}_{(4-\mathrm{n})}^{\prime}+\mathrm{MgX}
$$

onde $\mathrm{R}$ e R' = grupos orgânicos e n = varia de 1 a 3

Figura 5. Reação de alquilação de Grignard

A principal desvantagem da reação de "Grignard" é a formação simultânea de mono- e dissulfetos alquilados, que co-eluem com os OTs alquilados e, portanto, interferem na análise destes compostos por GC-FPD.

\section{Pré-concentração}

Entre os métodos de pré-concentração, a evaporação é o método mais popular. É preferido purgar o extrato com um fluxo brando de um gás inerte à rotaevaporação, que submete a amostra a condições desnecessárias de vácuo e aquecimento. No entanto, a evaporação do extrato derivado leva à pré-concentração simultânea de interferentes. Mesmo quando um detector seletivo é utilizado, até mesmo o solvente extraído sozinho e submetido à derivação com reagente de "Grignard" pode apresentar, após a pré-concentração, alguns picos ${ }^{67}$. 
Tabela 3. Concentrações de compostos butílicos de estanho detectadas em amostras de sedimento de diferentes países, obtidas por diferentes técnicas

\begin{tabular}{|c|c|c|c|c|c|c|}
\hline \multicolumn{7}{|c|}{ Concentração $\left[n g(\mathrm{Sn}) \mathrm{g}^{-1}\right.$ (peso seco)] } \\
\hline país & ano & técnica & MBT & DBT & TBT & referência \\
\hline Espanha & 1994 & GC-FPD & - & 482,0 & 994,0 & 71 \\
\hline Austrália & 1995 & AAS & - & - & $50-1350$ & 72 \\
\hline Polônia & 1997 & GC-FPD & - & - & $1800-2900^{\mathrm{a}}$ & 73 \\
\hline Japão & 1999 & GC-MIP-AED & 105 & 307 & 720 & 45 \\
\hline França & 2000 & GC-PFPD & $18-30$ & $<0,45-2,0$ & $3-12$ & 49 \\
\hline Suíça & 2000 & GC-FPD & $10-1300$ & $30-1900$ & $80-17000$ & 74 \\
\hline Índia & 2001 & GC-ICP-MS & $1,6-393$ & $1,3-394$ & n.d.-1280 & 75 \\
\hline Brasil & 2002 & GC-PFPD & $11,6-256,1$ & $8,2-703,8$ & $33,5-1388,2$ & 76 \\
\hline
\end{tabular}

${ }^{\mathrm{a}} \mathrm{MBT}+\mathrm{DBT}+\mathrm{TBT}$

\section{Purificação}

Extratos de amostras ricas em matéria orgânica geralmente carregam substâncias que são co-extraídas e que podem se depositar na coluna cromatográfica, influenciando a separação. O processo de purificação possui, portanto, a função de remover os principais interferentes da amostra, resultando em análises mais consistentes e reprodutíveis. O principal mecanismo envolvido no processo de purificação é o de adsorção, e os sólidos empregados, conhecidos como adsorventes, em geral são caracterizados por uma grande área superficial e pela presença de sítios específicos de adsorção ${ }^{69}$. A adsorção pode ser física, onde as moléculas são retidas na superfície do sólido simplesmente por forças atrativas entre as moléculas (forças de "Van der Waals"), ou química, onde as moléculas são retidas mais firme e especificamente por ligação química reversível na superfície do adsorvente como, por exemplo, pontes de hidrogênio. A propriedade básica da força de adsorção é a polaridade e, portanto, é o fator limitante do processo de dessorção. Se as moléculas adsorvidas são eluídas com solvente, o que comumentemente ocorre, estas podem ser extraídas da superfície do adsorvente por solubilização ou deslocadas dos sítios de adsorção por um solvente que fique fortemente adsorvido.

Os adsorventes mais utilizados são a sílica gel (com sítios ativos $\mathrm{Si}-\mathrm{OH}$ ), a alumina (com sítios ativos Al-OH) e o Florisil (preferido para matrizes bióticas com alto conteúdo lipídico $)^{20}$. Normalmente devem ser ativados por aquecimento antes do uso para dessorver a água ou outras moléculas que tenham sido adsorvidas da atmosfera.

\section{CENÁRIO MUNDIAL DA CONTAMINAÇÃO DO AMBIENTE MARINHO POR OTS}

A Tabela 3 mostra os níveis de concentração de compostos organoestânicos observados em amostras de sedimento de diversas partes do mundo, analisadas por diferentes técnicas cromatográficas. Existem evidências de que a contaminação por TBT tem diminuído após a implantação da legislação que proíbe o uso de tintas antiincrustrantes à base de TBT, em barcos menores de $25 \mathrm{~m}$ de comprimento, em países como Estados Unidos, Japão, Austrália, e a maioria da Europa. Os níveis ambientais do TBT vêm diminuindo na coluna d'água, nos sedimentos e nos organismos; além disso, há uma diminuição na ocorrência de má formação de conchas em ostras e de “imposex” em mexilhões. Os maiores níveis de poluição são encontrados apenas em áreas de grande atividade náutica, como portos e marinas $^{70}$.

De acordo com a Tabela 3, ainda são observados níveis altos de compostos butílicos de estanho (BTs), principalmente de TBT, em sedimentos de regiões onde existem grandes concentrações de bar- cos. Os valores de concentração de BTs encontrados no estudo feito na costa Paulista (Brasil) ${ }^{76}$ estão próximos daqueles encontrados em outros países e são indicativos de contaminação por tintas antiincrustrantes, já que os maiores valores foram observados em locais de maior movimento de barcos.

Portanto, há uma necessidade urgente de novos estudos para um mapeamento mais detalhado em toda a Costa Brasileira, para um diagnóstico preciso de toda a história da contaminação por TBT e seus derivados. O mapeamento é possível através da análise de "cores", ou "testemunhos de sedimentos", que são amostras coletadas em profundidade, em que, com a ajuda de estudos geoquímicos, é possível conhecer a idade da contaminação, o tempo de vida de cada composto e o mecanismo de degradação.

Estudos envolvendo os pesticidas OTs de uso comum principalmente no cultivo de frutas são ainda insipientes na literatura, provavelmente devido ao tempo bastante curto de persistência dos princípios ativos utilizados. No entanto, devido ao grande volume deste tipo de pesticida utilizado nas plantações de citrus da região central do estado de S. Paulo, caberia um diagnóstico da possível contaminação de cursos d'água da região, já que o maior impacto ecológico se dá sobre os organismos aquáticos.

\section{AGRADECIMENTOS}

Os autores agradecem à FAPESP e ao CNPq pelo auxílio financeiro.

\section{REFERÊNCIAS}

1. Patin, S. A.; Pollution and the Biological Resources of the Oceans, Butterworth Scientific: London, 1982.

2. Wensem, J. V.; Akkerhuis, G. A. J. M. J.; Straalen, N. M. V.; Pest. Sci. 1991, $32,307$.

3. Blunden, S. J.; Evans, C. J. Em The Handbook of Environmental Chemistry; Hutzinger, O., ed.; Springer-Verlag: Berlim, 1990, vol. 3.

4. Luijten, J. G. A. Em Organotin Compounds; Sawyer, A. K., ed.; Dekker: New York, 1987, vol. 3.

5. Crowe, A. J.; Appl. Organomet. Chem. 1987, 1, 143.

6. Champ, M. A.; Lowestein, F. L.; Ocean. Fall 1987, 30, 69.

7. Clare, A.; New Scientist 1995, february, 38.

8. Huggett, R. J.; Unger, M. A.; Seligmen, P. F.; Valkirs, A. O.; Environ. Sci. Technol. 1992, 26, 232.

9. Stebbing, A. R. D.; Mar. Pollut. Bull. 1985, 16, 383.

10. Sanyagina, N. A.; Makin, G. I.; Feshchenko, A. G.; Nesterova, G. N.; Shushunova, A. F.; Eurasian Soil Sci. 1993, 25, 108.

11. Souza Filho, M. F.; Suplicy Filho, N.; Sato, M. E.; Takematsu, A. P.; Pesquisa Agropecuária Brasileira 1994, 29, 1187.

12. Edge, V. E.; James, D. G.; J. Economic Entomology 1986, 79, 1477.

13. Flexner, J. L.; Westigard, P. H.; Croft, B.A..; J. Economic Entomology 1988, $81,1516$.

14. Lupan, S.; Anal. Chem. 1998, 71, 26. 
15. Laughlin, R. B.; Johannesen, R. B.; French, W.; Brinckman, F. E.; Guard, H.; Environ. Toxicol. Chem. 1985, 4, 343.

16. Cullen, W. R.; Eigendorf, G. K.; Nwata, B. U.; Takatsu, A.; Appl. Organomet. Chem. 1990, 4, 581.

17. Müller, M. D.; Anal. Chem. 1987, 59, 617.

18. Getzendanner, M. F.; Corbin, H. B.; J. Agric. Food Chem. 1972, $20,881$.

19. Müller, M. D.; Bosshardt, H. P.; Bull. Environ. Contam. Toxicol. 1987, 38, 627.

20. FAO/WHO Em Pesticide Residues in Food; Reported Sponsored Jointly by FAO and WHO, Rome, 1993.

21. Gray, A.; Dutton, A. J.; Eadsforth, C. V.; Pest. Sci. 1995, 43, 295.

22. Beaumont, A. R.; Newman, P. B.; Mar. Pollut. Bull. 1986, 17, 457.

23. Fent, K.; Crit. Rev. Toxicol. 1996, 26, 3.

24. Bryan, G. W.; Gibbs, P. E.; Huggett, R. J.; Curtis, L. A.; Bailey, D. S.; Dauer, D. M.; Mar. Pollut. Bull. 1989, 20, 458.

25. White, J. S.; Tobin, J. M.; Cooney, J. J.; Can. J. Microbiol. 1999, 45, 541.

26. Wong, P. T. S.; Chau, Y. K.; Kramar, O.; Bengert, G. A.; Can. J. Fish. Aquat Sci. 1982, 39, 483

27. Marsot, P.; Pelletier, É.; St-Louis, R.; Bull. Environ. Contam. Toxicol. 1995, 54,389 .

28. Kördel, W.; Stein, B.; Chemosphere 1997, 35, 191.

29. Fliedner, A.; Remde, A.; Niemann, R.; Schäfers, C.; Stein, B.; Chemosphere 1997, 35, 209.

30. Voulvoulis, N.; Scrimshaw, M. D.; Lester, J. N.; Appl. Organomet. Chem. 1999, 13, 135

31. Lyndon, A.; Mar. Pollut. Bull. 1999, 38, 847.

32. Vallee-Rehel, K.; Hoarau, B. M. P. A.; Guerin, P.; Langlois, V.; Langlois, J. Y.; J. Coat. Technol. 1998, 70, 55.

33. Jornal "O Estado de São Paulo”, 19 de fevereiro de 2000, seção de Ciência e Tecnologia, p. A16.

34. http://www. ortepa.org/pages/r1.htm, acessada em Abril 2002.

35. http://www.ortepa.org/pages/b5.htm, acessada em Abril 2002.

36. Gelmini, G. A.; Agrotóxicos, Legislação Básica, Fundação Cargill: Campinas, 1991, vol. 1 e 2.

37. Abalos, M.; Bayona, J. M.; Compaño, R.; Granados, M.; Leal, C.; Prat, M. D.; J. Chromatogr., A 1997, 788, 1.

38. Wu, J. C.; Mester, Z.; Pawliszyn, J.; J. Anal. At. Spectrom. 2001, 16, 159.

39. Kumar, U. T.; Dorsey, J. G.; Caruso, J. A.; Evans, E. H.; J. Chromatogr., A 1993, 655, 340.

40. Pobozy, E.; Glod, B.; Kaniewska, J.; Trojanowicz, M.; J. Chromatogr., A 1995, 718, 329.

41. Thomas, K. V.; Blake, S. J.; Waldock, M. J.; Mar. Pollut. Bull. 2000, 40, 739.

42. Encinar, J. R.; Gonzalez, P. R.; Alonso, J. I. G.; Sanz-Medel, A.; Anal. Chem. 2002, 74, 270.

43. Thomaidis, N. S.; Adams, F. C.; Lekkas, T. D.; Mikrochim. Acta 2001, 136, 137.

44. Gomez-Ariza, J. L.; Betrán, R.; Morales, E.; Giraldez, I.; Ruiz-Benitez, M.; Appl. Organomet. Chem. 1994, 8, 553.

45. Suzuki, T.; Kondo, K.; Uchiyama, M.; Murayama, M.; J. Agric. Food Chem. 1999, 47, 3886.
46. Vella, A. J.; Mintoff, B.; Axiak, V.; Sci. Total Environ. 2000, 258, 81

47. Waldock, M. J.; Waite, M. E.; Appl. Organomet. Chem. 1994, 8, 649

48. Carlier-Pinasseau, C.; Astruc, A.; Lespes, G.; Astruc, M.; J. Chromatogr., A 1996, 750, 317

49. Bancon-Montigny, C.; Lespes, G.; Potin-Gautier, M.; J. Chromatogr., A 2000, 896, 149.

50. Sullivan, J. J.; Torkelson, J. D.; Wekel, M. M.; Hollingworth, T. A.; Saxton, W. L.; Miller, G. A.; Danaro, K. W.; Uhler, A. D.; Anal. Chem. 1988, 60, 626.

51. Tolosa, I.; Merlini, J.; Debertrand, N.; Bayona, J. M.; Albaiges, J.; Environ. Toxicol. Chem. 1992, 11, 145.

52. Alzaga, R.; Bayona, J. M.; J. Chromatogr., A 1993, 655, 51.

53. Dachs, J.; Alzaga, R.; Bayona, J. M.; Anal. Quim. Acta 1994, 286, 319.

54. Wilken, R. D.; Kuballa, J.; Jantzen, E.; Fresenius J. Anal. Chem. 1994, 350, 77.

55. Rodriguez, I.; Santamaria, M.; Bollain, M. H.; Mejuto, M. C.; Cela, R.; J. Chromatogr., A 1997, 774, 379.

56. Aguerre, S.; Bancon-Montigny, C.; Lespes, G.; Potin-Gautier, M.; Analyst 2000, 125, 263

57. Millán, E.; Pawliszyn, J.; J. Chromatogr., A 2000, 873, 63.

58. Mester, Z.; Sturgeon, R. E.; Lam, J. W.; Maxwell, P. S.; Peter, L.; J. Anal. At. Spectrom. 2001, 16, 1313.

59. Abalos, M.; Bayona, J. M.; Quevauviller, P.; Appl. Organomet. Chem. 1998, 12,541

60. Ceulemans, M.; Adams, F. C.; Anal. Chim. Acta 1995, 317, 161.

61. Escobar, I. F.; Bayona, J. M., comunicação pessoal.

62. Schubert, P.; Fernandez-Escobar, I.; Rosenberg, E.; Bayona, J. M.; J. Chromatogr. A 1998, 810, 245.

63. Escobar, I. F.; Gilbert, M.; Messeguer, A.; Bayona, J. M.; Anal. Chem. 1998, 70, 3703

64. Curi, D.; Pardini, V. L.; Viertler, H.; Quim. Nova 1999, 22, 85.

65. Murray, R. W.; Chem. Rev. 1989, 89, 1187.

66. Morabito, R.; Massanisso, P.; Quevauviller, P.; Trends Anal. Chem. 2000, $19,113$.

67. Dirkx, W. M. R.; Lobinski, R.; Adams, F. C.; Anal. Chim. Acta 1994, 286 , 309

68. de la Calle-Guntiñas, M. B.; Scerbo, R.; Chiavarini, S.; Quevauviller, P.; Morabito, R.; Appl. Organomet. Chem. 1997, 11, 693.

69. Anderson, R.; Sample Pretreatment and Separation - Analytical Chemistry by Open Learning, John Wiley \& Sons: London, 1987.

70. Evans, S. M.; Leksono, T.; McKinnell, P. D.; Mar. Pollut. Bull. 1995, 30 , 14.

71. Cai, Y.; Alzaga, R.; Bayona, J. M.; Anal. Chem. 1994, 66, 1161.

72. Burt, J. S.; Ebell, G. F.; Mar. Pollut. Bull. 1995, 30, 723.

73. Kannan, K.; Falandysz, J.; Mar. Pollut. Bull. 1997, 34, 203.

74. Biselli, S.; Bester, K.; Huhnerfuss, H.; Fent, K.; Mar. Pollut. Bull. 2000, 40,233

75. Rajendran, R. B.; Tao, K.; Miyazaki, A.; Ramesh, R.; Ramachandran, S.; J. Environ. Monit. 2001, 3, 627

76. Godoi, A. F. L.; Montone, R. C.; Santiago-Silva, M.; J. Chromatogr., A 2003, 985, 205 